\title{
Clinical depression, posttraumatic stress disorder, and comorbid depression and posttraumatic stress disorder as risk factors for in-hospital mortality after coronary artery bypass grafting surgery
}

\author{
Tam K. Dao, PhD, ${ }^{\text {a,b,c }}$ Danny Chu, MD, ${ }^{\text {b,d }}$ Justin Springer, PhD, ${ }^{\text {b,c }}$ Raja R. Gopaldas, MD, ${ }^{\text {e }}$ \\ Deleene S. Menefee, PhD, ${ }^{\mathrm{b}, \mathrm{c}}$ Thomas Anderson, PhD, ${ }^{\mathrm{b}, \mathrm{c}}$ Emily Hiatt, BA, ${ }^{\mathrm{a}}$ and Quang Nguyen, $\mathrm{PhD}^{\mathrm{b}, \mathrm{c}}$
}

\begin{abstract}
Objective: The goal of this study was to examine the effect of clinical depression, posttraumatic stress disorder, and comorbid depression and posttraumatic stress disorder on in-hospital mortality after a coronary artery bypass grafting surgery. It is hypothesized that depression, posttraumatic stress disorder, and comorbid depression and posttraumatic stress disorder will independently contribute to an increased risk for in-hospital mortality rates after coronary artery bypass grafting surgery.
\end{abstract}

\begin{abstract}
Methods: We performed a retrospective analysis of the 2006 Nationwide Inpatient Sample database. The Nationwide Inpatient Sample database provides information on approximately 8 million US inpatient stays from about 1000 hospitals. We performed $\chi^{2}$ and unpaired $t$ tests to evaluate potential confounding group demographic and medical variables. Hierarchic logistic regression was used with forced order entry of depression, posttraumatic stress disorder, and comorbid depression and posttraumatic stress disorder.
\end{abstract}

Results: Deceased patients were more likely to have had depression (alive, $24.8 \%$; deceased, $60.3 \% ; P<.001$ ), posttraumatic stress disorder (alive, $13.4 \%$; deceased, $56.1 \% ; P<.001$ ), and cormorbid depression and posttraumatic stress disorder (alive, $7.8 \%$; deceased, $48.5 \% ; P<.001$ ). After adjusting for potential confounding factors, patients with depression (odds ratio, 1.24; 95\% confidence interval, 1.02-1.50), posttraumatic stress disorder (odds ratio, 2.09; 95\% confidence interval, 1.65-2.64), and comorbid depression and posttraumatic stress disorder (odds ratio, 4.66; 95\% confidence interval, 3.46-6.26) had an increased likelihood of in-hospital mortality compared with that seen in patients who were alive.

Conclusions: Two findings were noteworthy. First, depression, posttraumatic stress disorder, and comorbid depression and posttraumatic stress disorder are prevalent in patients undergoing coronary artery bypass grafting procedures. Second, depression, posttraumatic stress disorder, and comorbid depression and posttraumatic stress disorder increase the risk of death by magnitudes comparable with well-established physical health risk factors after coronary artery bypass grafting surgery. The implications for clinical practice and future directions are discussed. (J Thorac Cardiovasc Surg 2010;140:606-10)

Coronary artery disease (CAD) remains the leading cause of death in the United States, with more than 650,000 deaths registered in 2005. ${ }^{1}$ Patients with severe narrowing or blockage of the left main coronary artery or those with disease involving 3 coronary arteries are generally considered for coronary artery bypass grafting $(\mathrm{CABG})$ surgery. Research indicates that clinical outcomes, including length of hospital

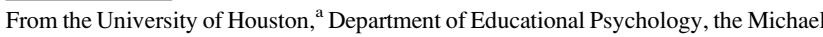
E. DeBakey Veterans Affairs Medical Center, ${ }^{\mathrm{b}}$ Mental Health Service Line, Baylor College of Medicine, ${ }^{c}$ and the Department of Psychiatry and Behavioral Sciences, ${ }^{d}$ Baylor College of Medicine, and the Division of Cardiothoracic Surgery, ${ }^{\mathrm{e}}$ Baylor College of Medicine, Houston, Tex, and USA Section of Adult Cardiac Surgery, The Texas Heart Institute at St. Luke's Episcopal Hospital, Houston, Tex. Supported by the Mental Illness Research, Education, and Clinical Center.

Disclosures: None.

Received for publication June 5, 2009; revisions received Sept 30, 2009; accepted for publication Oct 25, 2009; available ahead of print Jan 14, 2010.

Address for reprints: Tam K. Dao, PhD, University of Houston, Baylor College of Medicine, 2002 Holcombe Blvd, Houston, TX 77030 (E-mail: tdao@mail.coe.uh. edu).

$0022-5223 / \$ 36.00$

Copyright (c) 2010 by The American Association for Thoracic Surgery doi:10.1016/j.jtcvs.2009.10.046
}

stay, recurrent cardiac events, and in-hospital mortality, after a CABG surgery cannot be fully explained by traditional risk factors alone, such as age, sex, and medical comorbidities (eg, diabetes). ${ }^{2,3}$ As a result, the role of psychologic risk factors in explaining such outcomes has recently emerged.

To date, multiple longitudinal and cross-sectional studies have examined the association between negative CAD-related events and psychologic functioning, including depression. ${ }^{4}$ For example, preoperative depressive symptoms have been associated with cardiac readmissions up to 6 months ${ }^{2}$ and with higher rates of cardiac events between 1 and 8 years. ${ }^{3}$ Depressive symptoms also significantly predict mortality 2 to 5 years after CABG surgery, independent of medical and operative factors. ${ }^{3,5,6}$ Much of the research investigating the role of psychologic factors in explaining outcomes after a CABG surgery has focused on preoperative depression. In contrast to depression, the influence of other psychologic conditions, such as posttraumatic stress disorder (PTSD), on outcomes after CABG surgery has received less research attention. ${ }^{4}$ 


\section{Abbreviations and Acronyms \\ ANS = autonomic nervous system \\ CABG = coronary artery bypass grafting \\ $\mathrm{CAD}=$ coronary artery disease \\ HRV = heart rate variability \\ ICD-9-CM = International Classification of Dis- eases, 9th Revision, Clinical Modi- fication
OR $\quad=$ odds ratio
PTSD $=$ posttraumatic stress disorder \\ NIS $=$ Nationwide Inpatient Sample}

The paucity of research examining the PTSD-CABG outcome linkage creates a large gap in the literature for 2 reasons. First, pathophysiologic studies have associated both depression and PTSD with increased secretion of corticotropin-releasing factor. However, the increased secretion is associated with hypocortisolemia in patients with PTSD, whereas severe depression is associated with hypercortisolemia, which suggests that the pathophysiology of the 2 disorders might be distinct. ${ }^{7}$ Second, psychiatric comorbidities can act synergistically, potentially affecting cardiac prognosis adversely. The clinical implications of assessing for and treating only depression to the exclusion of PTSD might negatively affect cardiac outcomes.

The goal of this study was to examine the effect of clinical depression, PTSD, and comorbid depression and PTSD on mortality after a CABG surgery. It is hypothesized that depression, PTSD, and comorbid depression and PTSD will independently contribute to an increased risk for in-hospital mortality rates after a CABG surgery. Furthermore, given that both these disorders are linked to autonomic nervous system (ANS) dysregulation, it is hypothesized that comorbid depression and PTSD will have the greatest effect on mortality rates. If these hypotheses are supported, the case for ANS dysregulation as a potential mechanism responsible for the observed increase in mortality after a CABG surgery will be strengthened.

\section{MATERIALS AND METHODS Patient Population}

Institutional review board approval was obtained from the hospital facility, as well as from the authors' University Human Subject's Committee. The Nationwide Inpatient Sample (NIS) 2006 database was used to identify patients for this study. The NIS is a part of the Healthcare Cost and Utilization Project and contains about 8 million hospital discharges sampled from 1000 hospitals. The 2006 NIS database provides demographic, medical, and psychologic disease severity measures; postoperative complications; discharge disposition; and procedure and diagnostic codes classified according to the International Classification of Diseases, 9 th Revision, Clinical Modification (ICD-9-CM). ${ }^{8}$

By using the 2006 NIS database, 62,665 discharge records of patients who underwent primary CABG were identified. Independent variables in this study included age; sex (ie, female $=1$ and male $=0$ ); race (ie, white
$=1$ and other $=0$ ); median household income based on patient's ZIP code $(\$ 1-\$ 44,900=1$ and $\geq \$ 45,000=0)$; the Deyo, Cherkin, \& Ciol Comorbidity Index ${ }^{9}$; a depressive disorder diagnosis (ie, depression $=1$ and no depression $=0$ ); a PTSD diagnosis (ie, PTSD $=1$ and no PTSD $=0$ ); and a diagnosis of comorbid depression and PTSD (depression and PTSD $=1$ and no depression and PTSD $=0$ ). The Deyo, Cherkin, \& Ciol Comorbidity Index, which is adapted from the Charlson Comorbidity Index, ${ }^{10}$ is designed to capture comorbid conditions recorded in the inpatient setting using ICD-9-CM diagnosis and procedure codes. The Deyo, Cherkin, \& Ciol Comorbidity Index has been widely used in outcomes studies with administrative datasets as the principal data source.$^{11}$ It assesses comorbid medical conditions, such as myocardial infarction, congestive heart failure, peripheral vascular disease, cerebrovascular disease, dementia, chronic obstructive pulmonary disease, rheumatologic disease, mild liver disease, diabetes, diabetes with complications, hemiplegia or paraplegia, renal disease, malignancy, moderate-to-severe liver disease, metastatic solid tumors, and AIDS/HIV. The outcome variable in this study was in-hospital mortality. Because the NIS database contains only inpatient data, deaths occurring after hospital discharge were excluded from analyses.

\section{Data Analysis}

Data analyses proceeded in 2 sequential steps. First, $\chi^{2}$ and unpaired $t$ tests were performed to evaluate potential confounding group demographic and medical variables between the alive and deceased groups. Any variable that differed significantly between the 2 groups was used in subsequent regression models to test that variable's independent influence on medical outcomes after CABG surgery. Hierarchic logistic regression was used with forced order entry of depression, PTSD, and comorbid depression and PTSD. For the analyses, there were 3 data entry blocks in the hierarchy. Variables that have been shown in the literature to be associated with negative outcomes after CABG surgery were entered in the first block as potential confounding variables. In the second block these potential confounding variables and depression and PTSD were evaluated simultaneously. In the third block these variables were entered in with comorbid depression and PTSD. We decided to use dummy variable coding for comorbid depression and PTSD as opposed to an interaction term for ease of interpretation. It should be noted that entering comorbid depression and PTSD in the third block is similar to creating an interaction term. The results are identical to each other with respect to odds ratios (ORs).

\section{RESULTS}

Table 1 provides the demographic and clinical data for the aggregate and group-specific samples. The aggregate sample $(\mathrm{n}=62,665)$ consisted mostly of white $(85.3 \%)$ male $(71.3 \%)$ subjects with a mean age of 66.0 years (standard deviation, 10.87 years). Comorbid conditions ranged from $0.0 \%$ to $34.2 \%$ for AIDS/HIV and myocardial infarction, respectively. Of the sample, $25.9 \%$ had a diagnosis of depression, $14.7 \%$ had a diagnosis of PTSD, and $9.0 \%$ had a diagnosis of comorbid depression and PTSD. As indicated in Table 1, the alive and deceased groups differed significantly in terms of age; sex; race; median household income based on ZIP code; Deyo, Cherkin, \& Ciol Comorbidity Index; depression; PTSD; and comorbid depression and PTSD. Therefore these variables were included in subsequent regression models.

Table 2 contains results of hierarchic logistic regression analysis assessing the influence of depression, PTSD, and comorbid depression and PTSD on in-hospital mortality among patients undergoing CABG surgery. Block 1 
TABLE 1. Demographic and clinical data

\begin{tabular}{|c|c|c|c|c|}
\hline Demographics & Total $(n=62,656)$ & Alive $(\mathrm{n}=60,825)$ & Deceased $(n=1831)$ & $P$ value \\
\hline Age (y) & $66.0(10.87)$ & $65.8(10.86)$ & $70.64(10.57)$ & $<.001$ \\
\hline Male sex & $44,672(71.3 \%)$ & $43,534(71.6 \%)$ & $1133(61.9 \%)$ & $<.001$ \\
\hline White race & $37,561(85.3 \%)$ & $36,433(85.3 \%)$ & $1125(83.2 \%)$ & .031 \\
\hline Median household income based on ZIP code & & & & $<.001$ \\
\hline$\$ 1-\$ 35,900$ & $28,569(45.6 \%)$ & $27,615(45.4 \%)$ & $954(52.1 \%)$ & \\
\hline$\geq \$ 45,000$ & $34,087(54.4 \%)$ & $33,210(54.6 \%)$ & $877(47.9 \%)$ & \\
\hline Deyo, Cherkin, \& Ciol Comorbidity Index & $1.34(1.14)$ & $1.32(1.13)$ & $1.72(1.22)$ & $<.001$ \\
\hline Myocardial infarction & $21,445(34.2 \%)$ & $20,600(33.9 \%)$ & $844(46.1 \%)$ & $<.001$ \\
\hline Congestive heart failure & $13,907(22.2 \%)$ & $13,084(21.5 \%)$ & $820(44.8 \%)$ & $<.001$ \\
\hline Peripheral vascular disease & $4617(7.4 \%)$ & $4486(7.4 \%)$ & $130(7.1 \%)$ & .657 \\
\hline Cerebrovascular disease & $4177(6.7 \%)$ & $3984(6.5 \%)$ & $192(10.5 \%)$ & $<.001$ \\
\hline Dementia & $44(0.1 \%)$ & $43(0.1 \%)$ & $1(0.1 \%)$ & .798 \\
\hline COPD & $12,013(19.2 \%)$ & $11,572(19.0 \%)$ & $438(23.9 \%)$ & $<.001$ \\
\hline Rheumatologic disease & $814(1.3 \%)$ & $794(1.3 \%)$ & $20(1.1 \%)$ & .428 \\
\hline Peptic ulcer disease & $544(0.9 \%)$ & $523(0.9 \%)$ & $21(1.1 \%)$ & $<.001$ \\
\hline Mild liver disease & $200(0.3 \%)$ & $191(0.3 \%)$ & $9(0.5 \%)$ & 185 \\
\hline Diabetes & $18,938(30.2 \%)$ & $18,550(30.5 \%)$ & $387(21.1 \%)$ & $<.001$ \\
\hline Diabetes with chronic complications & $2518(4.0 \%)$ & $2438(4.0 \%)$ & $78(4.3 \%)$ & .589 \\
\hline Hemiplegia or paraplegia & $189(0.3 \%)$ & $174(0.3 \%)$ & $15(0.8 \%)$ & $<.001$ \\
\hline Renal disease & $51(0.1 \%)$ & $48(0.1 \%)$ & $3(0.2 \%)$ & .209 \\
\hline Malignancy & $837(1.3 \%)$ & $798(1.3 \%)$ & $39(2.1 \%)$ & .003 \\
\hline Moderate or severe liver disease & $43(0.1 \%)$ & $38(0.1 \%)$ & $5(0.3 \%)$ & .001 \\
\hline Metastatic solid tumor & $59(0.1 \%)$ & $55(0.1 \%)$ & $4(0.2 \%)$ & .078 \\
\hline AIDS/HIV & $0(0.0 \%)$ & $0(0.0 \%)$ & $0(0.0 \%)$ & - \\
\hline Depression & $16,215(25.9 \%)$ & $15,107(24.8 \%)$ & $1104(60.3 \%)$ & $<.001$ \\
\hline PTSD & $9202(14.7 \%)$ & $8170(13.4 \%)$ & $1027(56.1 \%)$ & $<.001$ \\
\hline Comorbid depression and PTSD & $5626(9.0 \%)$ & $4734(7.8 \%)$ & $888(48.5 \%)$ & $<.001$ \\
\hline
\end{tabular}

$C O P D$, Chronic obstructive pulmonary disease; $P T S D$, posttraumatic stress disorder.

indicates that age, sex, race, and Deyo, Cherkin, \& Ciol Comorbidity Index contributed to the prediction of group membership $\left(\chi^{2}[4,44,046]=432.6, P<.001\right)$. The Nagelkerke $R^{2}$ value, a comparable statistic to the $R^{2}$ value in linear regression, was 0.041 . The Hosmer-Lemeshow test, a test of the model's goodness of fit, produced a fail-to-reject decision $\left(\chi^{2}[8,44,046]=8.45, P=.391\right)$, a result consistent

TABLE 2. Hierarchic logistic regression analyses assessing depression and PTSD in predicting mortality in patients after CABG surgery

\begin{tabular}{lccc}
\hline \multicolumn{1}{c}{ Variable } & B & SE B & Odds ratio $(\mathbf{9 5} \%$ CI $)$ \\
\hline Constant & -6.324 & 0.212 & - \\
Age & $0.029 \dagger$ & 0.003 & $1.023(1.023-1.035)$ \\
Gender & $0.123^{*}$ & 0.060 & $1.131(1.005-1.274)$ \\
Race & -0.049 & 0.078 & $0.953(0.817-1.111)$ \\
Median household income & $0.212^{*}$ & 0.079 & $1.122(0.966-1.322)$ \\
Deyo, Cherkin, \& Ciol & $0.104 \dagger$ & 0.023 & $1.110(1.060-1.162)$ \\
$\quad$ & & & \\
$\quad$ Comorbidity Index & $0.215^{*}$ & 0.098 & $1.240(1.023-1.502)$ \\
Depression & $0.736 \dagger$ & 0.120 & $2.088(1.651-2.640)$ \\
PTSD & $1.539^{*}$ & 0.151 & $4.658(3.466-6.261)$ \\
Comorbid depression and PTSD & Note: Nagelkerke $R^{2}=0.041$ for block $1(P \mathrm{~s}<.001)$; change Nagelkerke $R^{2}=0.123$ \\
for block $2(P \mathrm{~s}<.001) ;$ change Nagelkerke $R^{2}=0.011$ for block $3(P \mathrm{~s}<.001) . P T S D$, \\
Posttraumatic stress disorder; $C A B G$, coronary artery bypass grafting; $S E$, standard er- \\
ror; $C I$, confidence interval. ${ }^{*} P<.05 . \dagger P<.001$.
\end{tabular}

with the assumption that the specified logistic model was correct. Independent significant predictors of mortality included age (OR, 1.05), sex (OR, 1.31), race (OR, 0.815), household income based on ZIP code (OR, 1.12), and the Deyo, Cherkin, \& Ciol Comorbidity Index (OR, 1.26). In block 2 depression and PTSD were entered and added significantly to the model with a change in $\chi^{2}$ value $\left(\chi^{2}[2,44,046]\right.$ $=1332.4, P<.001)$. The change in Nagelkerke $R^{2}$ value was 0.123 . Independent significant predictors of mortality included age (OR, 1.027); Deyo, Cherkin, \& Ciol Comorbidity Index (OR, 1.08); depression (OR, 2.51); and PTSD (OR, 5.34). In block 3 comorbid depression and PTSD was entered and added significantly to the model with a change in $\chi^{2}$ value $\left(\chi^{2}[1, \mathrm{n}=44,046]=119.8\right.$, $P<.001)$. The change in Nagelkerke $R^{2}$ value was 0.011 . Independent significant predictors of mortality included age (OR, 1.03); sex (OR, 1.13); Deyo, Cherkin, \& Ciol Comorbidity Index (OR, 1.11); depression (OR, 1.24); PTSD (OR, 2.08); and comorbid depression and PTSD (OR, 4.66).

\section{DISCUSSION}

There has been little systematic effort to investigate the role of other psychologic factors, such as PTSD, in explaining outcomes after CABG surgery. To our knowledge, there 
have been no studies that have simultaneously examined the comorbidity of depression and PTSD on mortality after a CABG outcome. This study found that depression, PTSD, and comorbid depression and PTSD independently and collectively contributed to increased in-hospital mortality. Initial analyses comparing deceased and alive patients revealed significant differences between groups in age, sex, race, depression, PTSD, and comorbid depression and PTSD diagnoses. The 2 groups were similar on preoperative medical comorbidities, as measured with the Deyo, Cherkin, \& Ciol Comorbidity Index. Deceased patients were more likely to be older, female, and nonwhite and to have depression, PTSD, or comorbid depression and PTSD diagnoses. These findings are preliminary, and further investigation is warranted.

Hierarchic logistic regression analyses indicated that age; sex; race; having a lower median household income based on ZIP code; Deyo, Cherkin, \& Ciol Comorbidity Index; depression; PTSD; and comorbid depression and PTSD were significant predictors of mortality. An examination of the Nagelkerke $R^{2}$ value change and the ORs suggests that depression, PTSD, and comorbid depression and PTSD increase the risk of death by magnitudes comparable with well-established physical health risk factors (eg, age) after CABG surgery. Older patients carried an increased risk for in-hospital mortality. A recent review of adverse events in CABG trials reported that the ORs associated with in-hospital mortality after CABG operations was $1.65 .{ }^{12}$ In this study the ORs found for age were similar to those reported by others. ${ }^{12}$ This finding establishes PTSD as an independent predictor of in-hospital mortality. Furthermore, depression-PTSD comorbidity was the strongest predictor, with the odds of mortality being 4 times higher in patients with both diagnoses than in those who did not have comorbid depression and PTSD.

Despite their unique presentations, one factor that has been understudied but appears to be central to the manifestation of both depression and PTSD is the role of the ANS. Three lines of evidence suggest that altered ANS activity in patients with depression and PTSD might account for the documented increased risk of mortality in patients with CAD.

First, both patients with depression and those with PTSD have been shown to have increased levels of plasma and urinary catecholamines (primarily norepinephrine) compared with levels seen in control subjects. ${ }^{13,14}$ This finding is particularly noteworthy given that plasma norepinephrine concentrations generally parallel the level of activity of the sympathetic nervous system and are highly correlated with sympathetic neural activity. ${ }^{14}$

Second, resting heart rate is higher in patients with depression and PTSD compared with that seen in control subjects. ${ }^{15,16}$ Both psychiatric conditions are also associated with an exaggerated heart rate response to physical and psychologic stressors in comparison with that seen in medically well patients ${ }^{12}$ and patients with CAD. ${ }^{17,18}$ Given that one of the primary functions of the ANS is to regulate heart rate through a reciprocal interaction of the sympathetic and parasympathetic nervous systems, the increased heart rate suggests altered ANS function.

A third line of evidence is based on previous studies that report decreased heart rate variability (HRV) among depressed patients compared with that seen among nondepressed patients. ${ }^{14}$ Over the last 2 decades, HRV has emerged as an important marker for examining autonomic flexibility, the continuous interplay between the parasympathetic and sympathetic influences, on heart rate. ${ }^{19}$ Increased HRV has been used as a marker of increased vagal activity and has been consistently associated with greater capacities to regulate stress, emotional arousal, and attention, ${ }^{20}$ whereas low HRV has been associated with excessive cardiac sympathetic modulation, inadequate parasympathetic modulation, or both. ${ }^{19}$ A number of studies have found HRV to be lower in both patients with depression and those with PTSD compared with that seen in control subjects. ${ }^{21}$ These findings strengthen the case for ANS dysregulation as a potential causal mechanism for increased mortality after CABG surgery.

Future studies should examine the effect of established ANS dysregulation markers in the presence of the diagnosis to examine the variance accounted for by the psychologic and physiopathologic variables. If ANS dysregulation accounts for the relation between emergent psychologic factors and medical outcomes, the implications for the preoperative assessment and treatment of both ANS dysregulation and psychologic comorbidities might be extensive.

The results of this study have implications for early assessment and integrated trauma-informed treatment of psychiatric conditions. Because comorbid psychiatric conditions, such as depression, are more frequently observed in cardiac care settings, ${ }^{22}$ clinicians (eg, cardiologists, nurses, and cardiothoracic surgeons) should accurately assess for comorbid psychiatric conditions, such as depression and PTSD, through the application of mental health screening instruments. Furthermore, given the challenges of cardiovascular care and the importance of early treatment for psychiatric conditions, patients with cardiac disease and comorbid depression or PTSD will benefit from the application of brief, evidence-based interventions for treating depression and PTSD.

As with many studies that use large administrative databases, there are several limitations to consider, the first of which involves the psychiatric diagnosis of patients. Our findings are based on the ICD-9-CM diagnosis codes selected for conditions associated with CABG, depression, and PTSD. Selected diagnosis codes might not capture all relevant cases and therefore could underestimate the actual prevalence of these conditions and affect other outcome 
measures of interest, such as length of stay. Detailed and precise information was not available to validate the diagnosis of CABG, depression, and PTSD. Thus these findings are preliminary, and further investigation is necessary. However, based on NIS technical documents, there were a number of quality control procedures that were used to assess the data quality for ICD-9-CM diagnoses. The reliability and validity of numeric data were assessed extensively by using internal and external forms of review. For instance, each discharge record was verified against established norms, when feasible, and values were compared with related elements. Furthermore, the NIS database was compared with 2 other national databases (ie, the National Hospital Discharge Survey and Medical Provider Analysis and Review) to assess for biases and was found to be quite similar to these 2 databases, particularly with regard to diagnosis and procedure codes.

Another inherent limitation of using a database is that it was not possible to establish the proximity of the diagnosis to the operation or to determine the course or severity of the diagnosis (eg, reactive vs chronic depression and mild vs severe depression). As one can imagine, a patient with chronic depression since adolescence is likely different from another patient with depression caused by a medical condition.

Also, although this study used ICD-9-CM codes to determine psychiatric diagnoses, patients in both the alive and deceased groups most likely had other comorbid diagnoses that were not identified; thus some caution is warranted in formulating interpretations pertaining to the true effects of depression and PTSD on mortality. However, despite the limitation inherent when using patients with comorbid diagnoses to examine one particular diagnostic group, this limitation can also be viewed as addressing the ecologic validity of the study, given that a large number of patients in the clinical settings are diagnosed with co-occurring disorders. ${ }^{23}$ Despite these limitations, the current results do provide additional support for the importance of presurgical screening for depression and PTSD, as well as the development and implementation of short-term treatments for depression and PTSD that might facilitate better outcomes after CABG operations.

\section{References}

1. Centers for Disease Control and Prevention (CDC). Web-based injury statistics query and reporting system (WISQARS). National Center for Injury Prevention and Control, CDC (producer). Available at: www.cdc.gov/ncipc/wisqar/default. htm. Accessed Dec 3, 2009.

2. Saur C, Granger BB, Muhlbaier H, et al. Depressive symptoms and outcome of coronary artery bypass grafting. Am J Crit Care. 2001;10:4-10.

3. Blumenthal JA, Lett HS, Babyak MA, et al. Depression as a risk factor for mortality after coronary artery bypass surgery. Lancet. 2003;362:604-9.

4. Oxlad M, Stubberfield J, Stuklis R, Edwards J, Wade TD. Psychological risk factors for cardiac-related hospital readmission within 6 months of coronary artery bypass graft surgery. J Psychosom Res. 2006;61:775-81.

5. Burg MM, Benedetto C, Rosenberg R, et al. Presurgical depression predicts medical morbidity 6 months after coronary artery bypass graft surgery. Psychosom Med. 2003;65:111-8.

6. Burg MM, Benedetto C, Soufer R. Depressive symptoms and mortality two years after coronary artery bypass graft surgery (CABG) in men. Psychosom Med. 2003;65:508-10.

7. Lyons D, McLoughlin DM. Recent advances: psychiatry. BMJ. 2001;323: 1228-31.

8. ICD-9-CM. International Classification of Diseases, 9th Revision, Clinical Modification. 6th ed. Los Angeles: Practice Management Information Corporation; 2006.

9. Deyo RA, Cherkin DC, Ciol MA. Adapting a clinical comorbidity index for use with ICD-9-CM administrative databases. J Clin Epidemiol. 1992;45:613-9.

10. Charlson ME, Pompei P, Ales KL, et al. A new method of classifying prognostic comorbidity in longitudinal studies: development and validation. J Chronic Dis. 1987;40:373-83.

11. Needham D, Scales D, Laupacis A, Pronovost P. A systematic review of the Charlson comorbidity index using Canadian administrative databases: a perspective on risk adjustment in critical care research. J Crit Care. 2005;20:12-9.

12. Nalysnyk L, Fahrbach K, Reynolds M, Zhao S, Ross S. Adverse events in coronary artery bypass graft $(\mathrm{CABG})$ trials: a systematic review and analysis. Heart. 2003;89:767-72.

13. Yehuda R, Siever LJ, Teicher MH, et al. Plasma norepinephrine and 3-methoxy4-hydroxyphenylglycol concentrations and severity of depression in combat posttraumatic stress disorder and major depressive disorder-a chronobiological analysis. Biol Psychiatry. 1998;44:56-63.

14. Lake CR, Pickar D, Ziegler MG, Lipper S, Slater S, Murphy DL. High plasma NE levels in patients with major affective disorder. Am J Psychiatry. 1982;139: 1315-8.

15. Siever L, Davis K. Overview: toward a dysregulation hypothesis of depression. Am J Psychiatry. 1985;142:1017-31.

16. Veith RC, Lewis N, Linares OA, et al. Sympathetic nervous system activity in major depression. Arch Gen Psychiatry. 1994;51:411-22.

17. Carney RM, Rich MW, teVelde A, Saini J, Clark K, Freedland KE. The relationship between heart rate, heart rate variability and depression in patients with coronary artery disease. J Psychosom Res. 1988;32:159-64.

18. Carney RM, Saunders RD, Freedland KE, Stein P, Rich MW, Jaffe AS. Association of depression with reduced heart rate variability in patients with coronary heart disease. Am J Cardiol. 1995;76:562-4.

19. Appelhans BM, Luecken LJ. Heart rate variability as an index of regulated emotional responding. Rev Gen Psychol. 2006;10:229-40.

20. Bornstein MH, Suess PE. Physiological self-regulation and information processing in infancy: cardiac vagal tone and habituation. Child Dev. 2002;71:273-87.

21. Imaoka K, Inoue H, Inoue Y, Hazama H, Tanaka T, Yamane N. R-R intervals of ECG in depression. Folia Psychiatr Neurol Jpn. 1985;39:485-8.

22. Rumsfeld JS, Ho M. Depression and cardiovascular disease. Circulation. 2005; 111:250-3.

23. Kaplan HI, Sadock BJ. Synopsis of psychiatry. 8th ed. Baltimore: Lippincott Williams \& Wilkins; 1998. 